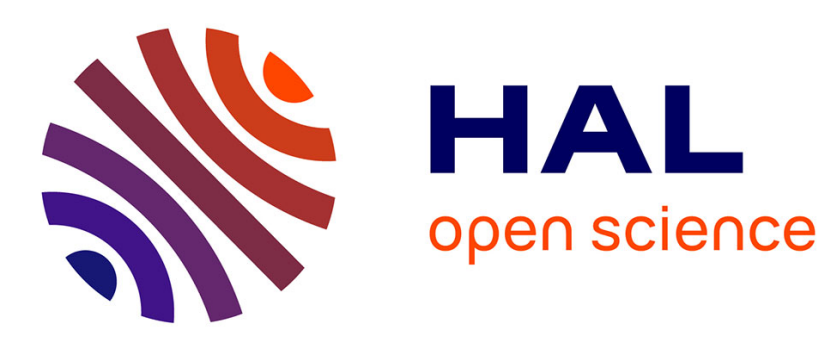

\title{
Rheological and mechanical properties of silica colloids: from Newtonian liquid to brittle behaviour
}

\author{
Erika Di Giuseppe, Anne Davaille, Eric Mittelstaedt, Marc Louis Maurice
}

François

\section{- To cite this version:}

Erika Di Giuseppe, Anne Davaille, Eric Mittelstaedt, Marc Louis Maurice François. Rheological and mechanical properties of silica colloids: from Newtonian liquid to brittle behaviour. Rheologica Acta, 2012, 51, pp.451-465. 10.1007/s00397-011-0611-9 . hal-01007258

\section{HAL Id: hal-01007258 \\ https://hal.science/hal-01007258}

Submitted on 24 Jun 2019

HAL is a multi-disciplinary open access archive for the deposit and dissemination of scientific research documents, whether they are published or not. The documents may come from teaching and research institutions in France or abroad, or from public or private research centers.
L'archive ouverte pluridisciplinaire HAL, est destinée au dépôt et à la diffusion de documents scientifiques de niveau recherche, publiés ou non, émanant des établissements d'enseignement et de recherche français ou étrangers, des laboratoires publics ou privés. 


\title{
Rheological and Mechanical properties of Hard-Sphere Silica Colloids : from Newtonian liquid to brittle behaviour
}

\author{
Erika Di Giuseppe ${ }^{1}$, Anne Davaille ${ }^{1}$, Eric Mittelstaedt ${ }^{1}$, Marc François ${ }^{1}$ \\ Laboratoire FAST (CNRS / Univ. Paris 6 / Univ. Paris 11), Bat. 502, Rue du Belvédère, Campus Universitaire, 91405 ORSAY \\ cedex, France, digiuseppe@fast.u-psud.fr
}

\begin{abstract}
Rheological and mechanical properties of aqueous mono-disperse hard-sphere silica suspensions (Ludox® are investigated as a function of particle volume fraction ( $\phi_{p}$ ranging from 0.22 to 0.51 ), using shear rate tests, oscillatory methods, indentation and ultrasonic technique. Four regimes are identified with increasing particle content; they are related to the existence and behaviour of the electrical double layer (EDL) around each particle. Semi-diluted suspensions $\left(0.22 \leq \phi_{p} \leq 0.30\right)$, which do not flocculate due to the EDL, show Newtonian behavior (I). At higher particle contents, the suspensions exhibit two viscoelastic (VE) shear thinning regimes separated by a critical particle volume fraction. It discriminates semi-concentrated fluids (II, $0.31 \leq \phi_{p} \leq 0.35$ ), in which particles form aggregates of quickly increasing size, from concentrated strain sensitive samples with paste-like aspect and high elastic modulus (III, $0.35 \leq \phi_{p} \leq 0.47$ ). Regime II can be explained by a sudden partial collapse of the EDL as it is progressively squeezed with increasing $\phi_{p}$. At higher particle concentration, the suspensions undergo a glass transition and behave as an elastic-brittle solid (IV, $\left.\phi_{p}=0.51\right)$.
\end{abstract}

\section{Introduction}

Suspensions of hard particles are one of the most important classes of colloidal suspensions (Mason and Weitz 1995). How hard-sphere colloidal solutions dry and aggregate is of great interest in many industrial applications (Dames et al 2001) including paints, coatings, colloidal ceramics, milk products (Dickinson 1992), and electronics manufacturing. As drying proceeds, the solutions pass through different stages from purely viscous to elastic and brittle. Such materials also provide

Send offprint requests to: Erika Di Giuseppe good analogs to planetary rocks whose behavior, as temHowlertature decreases, changes from viscous flow to elastic/brittle deformation with increasingly non-linear strain localization. Gauthier et al $(2007,2010)$ used Ludox®silica hard-sphere colloidal solutions to investigate the formation and the dynamics of cracks in 3-dimensional problems. The apparent hierarchy and the self-organized patterns of the cracks induced by drying show similarities with fracture networks in muds, permafrost, sediments, paint, concrete, and cooling of lava flows and magma chambers. Pauchard (2006) focused on the delamination process occurring during the drying of a thin Ludox®layer coating a substrate, a situation encountered for exemple in the splitting of cells sheets in embryogenesis, or in mud drying. Recently Di Giuseppe and Davaille (2010) also used Ludoxß)solutions to model convection in planetary mantles.

However, although the phenomenology of these processes has gained significant insights, quantitative predictions for the industrial or natural systems are still lacking. This is largely due to our imperfect knowledge of the rheology of these solid particles suspensions as they evolve from Newtonian to brittle. The difficulty here is that their macroscopic behaviour does not only depend on the volume fraction, $\phi_{p}$, of particles (Jeffrey and Acrivos, 1976), but is strongly dependent on several microscopic factors such as the surface electrical charge of the individual particles (Russel, 1978; van de Ven 1989; Allain et al, 1993), the size and the shape of the particles (Jeffrey and Acrivos 1976; Yanez et al 1999), their size distribution (Jeffrey and Acrivos, 1976), the formation of aggregates and their network structure (Lin et al, 1989; Prasher et al 2006; Chen et al 2007; Chevalier et al 2009), and large-scale factors such as the temperature, T, (e.g. Birdi 1997; Jones et al. 1991; Mason and Weitz 1995; Mattsson et al. 2009).

We study here the rheology of Ludox $₫$ silica nanoparticles suspensions. In the absence of evaporation, the suspensions are stable due to competition of the attractive van der Waals forces and the electrostatic repulsion be- 
tween the nanometer-size silica particles (Gauthier et al. 2007). The silica particles are hard spheres and numerous models have been developed to predict the viscosity of neutral hard-spheres dispersion as the particle content increases (e.g. Einstein 1911, Brinkman and Roscoe, 1952, Batchelor 1970, Krieger and Dougherty 1959, Quemada 1977). However, charges on the surface of the particles can cause an increase of the viscosity, due to the resistance against deformation and flow of the electronic cloud around each particle (e.g. Russel, 1978, 2009; Sherwood, 1980; Allain et al, 1993; Sherwood et al, 2000; Quemada and Berli, 2002; Berli et al, 2005). Moreover, they can promote particle aggregation, which also tends to increase the dispersion viscosity (e.g. Tsenoglou, 1990; Aubry et al, 1998; Chen et al, 2007; Chougnet et al, 2007; Bullard et al, 2009; SantamaraHolek and Mendoza, 2010). Besides, aggregation also results in the creation of a solid squeleton, so that solid behaviour appears (e.g. Lin et al, 1989; Grant and Russel, 1993; Mason and Weitz, 1995; Rueb and Zukoski, 1997; Channel and Zukoski, 1997). At low particle volume fraction (i.e. $\leq 22 \%$ ), Ludox@dispersions have been extensively used to examine the gelation and rheological properties of nanoparticles suspensions when their ionic composition was changed. Allain et al (1993) varied the suspension ionic strength by adding salt and the particle fraction by diluting the suspensions. The measured viscosity values are higher that the classic hard-sphere model predictions but can be rescaled on an universal curve, with coefficient depending on the electrostatic repulsion between the particles. Pauchard et al (1999) further showed that the gelation time decreased strongly with increasing ionic strength: salt effectively screens the electrostatic repulsion, favoring the gelation of the suspensions. Drabarek et al (2002) studied the effects of $\mathrm{pH}$ and particle size on structural evolution during gelation of Ludox $囚$ HS-40 and TM-50. They found that viscoelastic behaviors of the suspensions during gelation are similar but the microstructural properties differ significantly if the $\mathrm{pH}$, particle volume fraction, or particle size are varied. Trompette and coauthors showed, by means of dynamic measurements, how the nature of the electrolyte, such as a structure-breaker ammonium counterion or a structure-maker sodium counterion, and their concentration strongly affect the kinetics of gelation (Trompette and Meireles, 2003), and the strength of the gelled network (Trompette and Clifton, 2004) by varying significantly the critical coagulation value. Park et al (2005) found that, despite the fact that a positively charged surface of Ludox@suspension accelerates gelation, the stabilizing sodium ions have to be removed to achieve a high gel strength. Madeline et al (2007) studied the restructuring processes when low volume fraction Ludox@suspensions are dewatered by osmotic pressure and compressed to the point where the colloid aggregates connected to each other and built a macroscopic network. Cao et al (2010) followed the fractal growth of the clusters'size and the evolution of the elastic modulus during gelation, as a function of the initial salt concentration. On the other hand, Zarzycki (1988) provided the first determinations of critical stress intensity factors and of fracture surface energy of the crust forming on thick layers of wet Ludox囚gels dried from above. Pauchard (2006) extended these results to the Young's modulus by means of indentation tests. Finally, Malzbender et al (2002) determined the elastic modulus and hardness of dried silica-filled thin polymeric coatings.

So, Ludoxßproperties are reasonably known for low $\left(\phi_{p} \leq 22 \%\right)$ and for high $\left(\phi_{p} \geq 50 \%\right)$ particle volume fractions, but data is lacking for intermediate values of $\phi_{p}$. Therefore, the aim of the present work is to measure and analyze the effects of $\phi_{p}\left(22 \% \leq \phi_{p} \leq 58 \%\right)$ and the temperature $T\left(5 \leq T \leq 45^{\circ} \mathrm{C}\right)$ on the rheological and mechanical properties of Ludox $\AA$ HS- 40 . The samples' preparation is detailed in section 2 . The commercial suspensions are first studied in the original form supplied by the manufacturer, and then with increasing particle volume-fraction achieved by drying. We use a variety of rheological measurement techniques including controlled shear rate tests and oscillatory experiments for fluid or pasty samples (section 3). Indentation and ultrasonic methods are used for solid samples (section 4). The colloidal suspension evolves from a viscous Newtonian fluid to a gel-like state and finally to a brittle solid with increasing particle volume fraction. These macroscopic rheological variations are discussed in terms of interactions among the nanoparticles and of cluster (aggregate) formation (section 5).

\section{Fluids and samples}

\subsection{Material}

We used an aqueous suspension of mono-disperse silica particles, (Ludox@HS-40, 40 wt\% solid fraction) commercially available from Sigma-Aldrich (www.sigmaaldrich.com/). Diffusion measurements give a particle diameter $a=$ $15 \pm 2 \mathrm{~nm}$ (Gauthier et al, 2007). The silica particles are dispersed in an aqueous phase which also contains $\mathrm{Na}^{+}$, $\mathrm{OH}^{-}(p H=9.7)$ and silicate ions. The silica surfaces carry a high density of $\mathrm{SiO}^{-}$groups, which are compensated for by $\mathrm{Na}^{+}$counterions in the aqueous phase. The layers of counterions that surrounds neighbouring particles overlap, producing strong interparticle repulsions (Madeline et al, 2007). This ensures that the suspension, with a solid volume fraction $\phi_{p}=22 \%$, does not floculate. Small angle neutron scattering (SANS) experiments show a strong peak corresponding to the regular spacing of repelling silica particles. The average distance between particle centers determined from the peak position is $19 \mathrm{~nm}$ (Madeline et al, 2007). The density of 


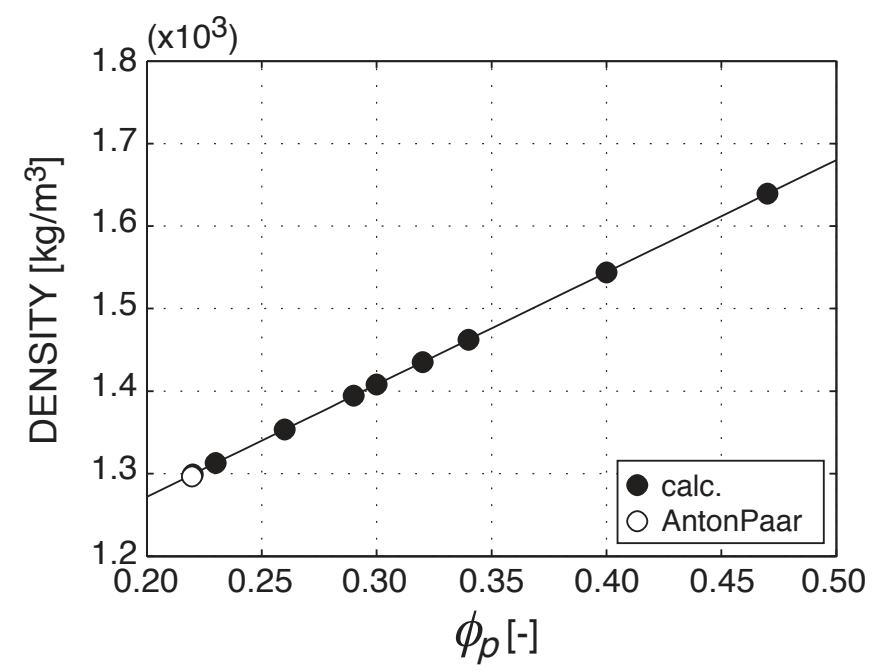

Fig. 1 Density of Ludox®HS-40 suspensions at $22^{\circ} \mathrm{C}$ for different volume particle concentrations. Black symbols show the calculated values of density using equations (1) and (2), and the white symbol shows the density measured with the DMA 5000 densimeter.

$\left.\begin{array}{|l|c|c|c|}\hline \phi_{p} & \begin{array}{c}\rho \\ -\end{array} & p H & \begin{array}{c}r_{\text {eff }} \\ \left(10^{3} \mathrm{~kg} / \mathrm{m}^{3}\right)\end{array}\end{array}\right)$

Table 1 Particle volume fraction, density, $\mathrm{pH}$, Debye length, and effective particle radius of the analyzed Newtonian samples at $22^{\circ} \mathrm{C}$.

Ludox@HS-40 has been measured with a Anton Paar DMA 5000 and is $\rho_{0}=1.295 \pm 0.001 \mathrm{~g} / \mathrm{cm}^{3}$ at $25^{\circ} \mathrm{C}$.

\subsection{Sample preparation}

In order to investigate how $\phi_{p}$ affects the rheological properties of silica suspensions, several samples were prepared by slowly drying the Ludox@)commercial solution. A known volume of Ludox@in a glass beaker (typically $300 \mathrm{~mL}$ ) is weighed with a high accuracy balance, and heated on a hot plate at a constant, low temperature (i.e. $T=40^{\circ} \mathrm{C}$ ) while being slowly stirred to maintain a homogeneous suspension. Drying time varies between samples from 2 to 8 days depending upon the desired particle concentration.

The particle volume fraction $\phi_{p}$ and suspension density $\rho$ are evaluated from the measurements of the initial (before drying) and the final (after drying) weights, $m_{0}$ and $m_{f}$, respectively. The accuracy of the mass measurements is $\pm 0.0001 \mathrm{~g}$. Given that (1) only the solvent evaporates from the sample during drying, (2) the volume occupied by hard particles does not change, (3) the solvent and particle densities, are respectively $\rho_{s}=1.0$ $\mathrm{g} / \mathrm{cm}^{3}$ and $\rho_{p}=2.36 \mathrm{~g} / \mathrm{cm}^{3},(4)$ the solvent volume fraction is $\phi_{s}=1-\phi_{p}$ and (5) the solid weight fraction of the Ludox@HS-40 initially is 0.40 . Then for each dried suspension, the silica weight fraction is $x_{s}=0.40 m_{0} / m_{f}$ and the solid volume fraction is:

$$
\phi_{p}=\frac{x_{s}}{x_{s}+\left(1-x_{s}\right) \frac{\rho_{p}}{\rho_{s}}}
$$

The uncertainty on $\phi_{p}$ is \pm 0.005 . The density of the solution is then:

$$
\rho=\rho_{s}\left(1+\phi_{p}\left(\frac{\rho_{p}}{\rho_{s}}-1\right)\right)
$$

Following the above drying procedure, we created eight solutions from Ludox $囚 \mathrm{HS}-40$ with $\phi_{p}$ ranging from 0.22 (the original suspension provided by the manufacturer) to 0.47 (Tables 1 and 2). Samples with $\phi_{p}=0.32$ 0.34 present a turbid aspect, while samples with lower or higher particle content were transparent. Measurements of the $\mathrm{pH}$ of the low $\phi_{p}$ solutions were performed by using a pHTestr10 (EUTECH Instruments, www.eutechinst.com) whose $\mathrm{pH}$ range is between 4 and 10, with an accuracy of 0.01 (Table 1). As water evaporates and the particle content increases, we notice a weak decrease of the solution $\mathrm{pH}$.

To produce solid samples, $100 \mathrm{ml}$ of Ludox $囚 \mathrm{HS}-40$ is poured into a glass tank measuring $15 \times 10 \times 1.5 \mathrm{~cm}^{3}$ which is placed next to an open tank of water (to maintain a high-humidity environment, $>45 \%$ ) within a closed Plexiglas container. Then it is allowed to dry at room temperature $\left(21^{\circ} \mathrm{C}\right)$ for $\sim 4$ weeks. During this process, the sample breaks into flat solid fragments of sizes ranging from $\sim 1$ to $4 \mathrm{~cm}$ with a nearly uniform thickness of $\sim 2.25 \mathrm{~mm}$. The density of the dried sample $\left(\rho_{\text {dried }}\right.$ $=1.685 \pm 0.005 \mathrm{~g} / \mathrm{cm}^{3}$ ) was measured by a pycnometric balance in demineralized water (the measurement time is kept short enough so that the specimen properties are not affected). According to equation (2), the particle volume fraction in the solid is $0.51 \pm 0.05$, much smaller than the maximum close packing value $\left(\phi_{C P}=0.74\right)$ as well as smaller than the random close packing value for solid spheres $\left(\phi_{R C P} \sim 0.63\right)$. This suggests that the material microstructure is disordered, and probably results from random packing of particle aggregates. This consideration, as well as the curved shape of the cracks, show that the structure is not a monocrystal.

\section{Rheological measurements}

Rheometric measurements are performed on a Physica MCR 501 (Anton Paar, www.anton-paar.com/) rheometer, with an accessible shear rate range from $10^{-6}$ to $3.10^{3} \mathrm{~min}^{-1}$. Two different geometries are used in the 


\begin{tabular}{|c|c|c|c|c|c|c|c|c|c|c|c|}
\hline$\phi_{p}$ & $\begin{array}{c}\rho \\
10^{3} \mathrm{~kg} / \mathrm{m}^{3}\end{array}$ & $\eta \propto \dot{\gamma}^{n}$ & $\begin{array}{c}\sigma_{Y} \\
(\mathrm{~Pa})\end{array}$ & $\begin{array}{c}\sigma_{f} \\
(\mathrm{~Pa})\end{array}$ & $\begin{array}{l}\gamma_{L} \\
(\%)\end{array}$ & $\begin{array}{c}\sigma_{L} \\
(\mathrm{~Pa})\end{array}$ & $\begin{array}{l}\sigma_{f a} \\
(\mathrm{~Pa})\end{array}$ & $\begin{aligned} G^{\prime} & \sim \omega^{a} \\
G^{\prime \prime} & \sim \omega^{b}\end{aligned}$ & $\begin{array}{l}\tau_{M} \\
(\mathrm{~s})\end{array}$ & $\begin{array}{l}G_{\infty} \\
(\mathrm{Pa})\end{array}$ & $\begin{array}{c}D_{o} \\
\left(10^{-9} \mathrm{~m}\right)\end{array}$ \\
\hline & & $\overline{\mathrm{FT}}$ & FT & FT & AST & $\mathrm{AST}$ & AST & AST & FST & FST & FST \\
\hline 0.32 & 1.435 & $\mathrm{n}=-0.69$ & 7.6 & 320 & 12.6 & 36.0 & - & $\begin{array}{l}a=1.6 \\
b=1.0\end{array}$ & 0.003 & $1100-2600$ & $4.2-1.8$ \\
\hline 0.34 & 1.462 & $\mathrm{n}=-0.77$ & 56.0 & 340 & 2.5 & 85.0 & - & $\begin{array}{l}a=2.0 \\
b=1.0\end{array}$ & 0.051 & $1.0-1.2 \times 10^{4}$ & $0.49-0.42$ \\
\hline 0.40 & 1.544 & $\mathrm{n}=-0.91$ & - & 1470 & 2.5 & 1000 & 1810 & $\mathrm{G}$ plateau & $50-500$ & $8.1 \times 10^{4}$ & 0.22 \\
\hline 0.47 & 1.639 & $\mathrm{n}=-0.97$ & - & 3750 & 0.8 & 1220 & 2490 & $\mathrm{G}$ plateau & $250-10^{4}$ & $2.5 \times 10^{5}$ & 0.20 \\
\hline
\end{tabular}

Table 2 Values of the different measurements for the samples with $0.32 \leq \phi_{p} \leq 0.47$ at $22^{\circ} \mathrm{C}$ (see text for the symbols). FT, AST and FST designate the type of rheology test (FT: flow test; AST: amplitude sweep test; FST: frequency sweep test)

measurements: a coaxial cylinder (CC) and a cone-andplate $(\mathrm{CP})$ with a truncated cone tip geometry. Following Mezger (2002), CC is used for low-viscosity fluids $\left(0.22 \leq \phi_{p} \leq 0.34\right)$ and $\mathrm{CP}$ for more pasty, higher viscosity samples $\left(0.40 \leq \phi_{p} \leq 047\right)$. The temperature control, in both geometries, is ensured by a Peltier element, providing rapid heating and cooling with an accuracy of $0.01{ }^{\circ} \mathrm{C}$. The coaxial cylinder, CC27, has a measuring bob diameter of $26.659 \mathrm{~mm}$, a measuring cup diameter of $28.918 \mathrm{~mm}$, and a gap length of $40 \mathrm{~mm}$. The CP measuring system, CP-50-0.5, has a cone angle of $0.484^{\circ}$ and a cone to lower plate distance of $\sim 25 \mu \mathrm{m}$. The coneto-plate distance is set manually and verified throughout the test by the rheometer, which notifies the user of large changes to the specified distance. After sample emplacement, excess material is removed from the edges of the cone to eliminate edge effects. Profiled surfaces are not required for high $\phi_{p}$ samples because of their adhesive behavior. A solvent trap, filled with water, minimizes the evaporation of the solvent in both adopted geometries. We waited $\sim 30$ min before each test, to allow the suspension microstructure to relax after pouring. We examine the properties of each prepared solution in a series of rheological tests including a controlled shear-rate test, and two dynamic sweep (oscillatory) tests, an amplitude sweep test and a frequency sweep test. For each solution, a new sample is taken for each test

\subsection{Dependence on the shear-rate}

The controlled shear rate test measures the viscous behavior of the samples in sol-state. In this test, the shear strain rate, $\dot{\gamma}$, is varied between $10^{-1}$ and $10^{3} \mathrm{~s}^{-1}$ in a logarithmic ramp profile, to ensure the achievement of steady state flow, while temperature is held constant $\left(T=22^{\circ}\right.$ C; Fig. 2 and Fig. 3$)$.

Both the viscosity $\eta$ and the non-newtonian character of the fluid increase dramatically with increasing $\phi_{p}$ (Fig. 2). Samples with $\phi_{p} \leq 0.30$ behave as a viscous Newtonian fluid over the entire range of shear rates investigated (Fig. 2). When $\phi_{p}>0.30$, the samples become shear thinning (Fig. 2), and an apparent yield stress $\sigma_{Y}$ can be detected at low shear rate (Fig. 3). However,

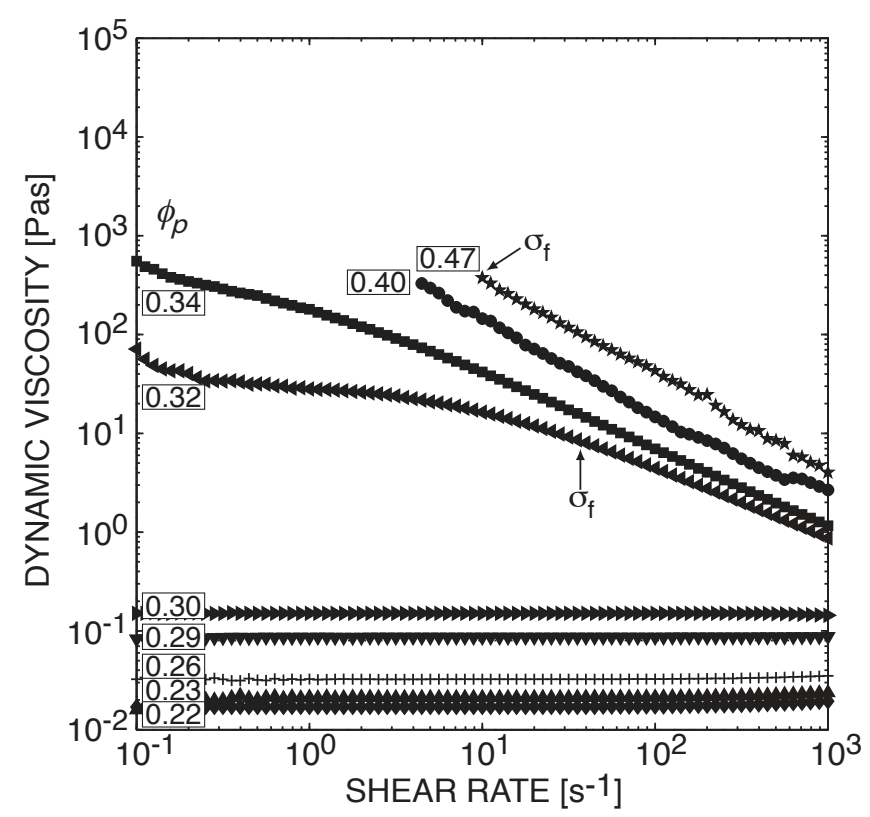

Fig. 2 Viscosity $\eta$ obtained by flow test, versus shear rate $\dot{\gamma}$ for different particle volume fractions. Each symbol represents a different $\phi_{p}$ value, indicated in a square for each set of measurements. $\sigma_{f}$ corresponds to the limit above which the shear-thinning exponent $n$ is constant $\left(\eta \propto \dot{\gamma}^{n}\right)$.

the rheology cannot be described with a Hershel-Buckley model because the shear-thinning character of the fluid changes at high shear: on Fig. 3, the slope of the flow curve, although always positive, decreases at high shear rate. When $\phi_{p} \geq 0.40$, measurements are erratic at low shear rate (Fig.2), which probably is the signature of small fractures in the pasty samples. But at high shear rate values $\left(\dot{\gamma}>5 \mathrm{~s}^{-1}\right)$, we see again the shear-thinning behaviour (Fig. 2), as the material reorganizes itself to allow shear. We define $\sigma_{f}$ as the stress above which a shear-thinning behaviour with a constant power law (i.e. $\eta \propto \dot{\gamma}^{n}$ ) prevails (Fig.2 and Fig.3).

\subsection{Temperature dependence}

To investigate the influence of temperature and to check for a possible effect of solvent evaporation on $\eta$, we per- 


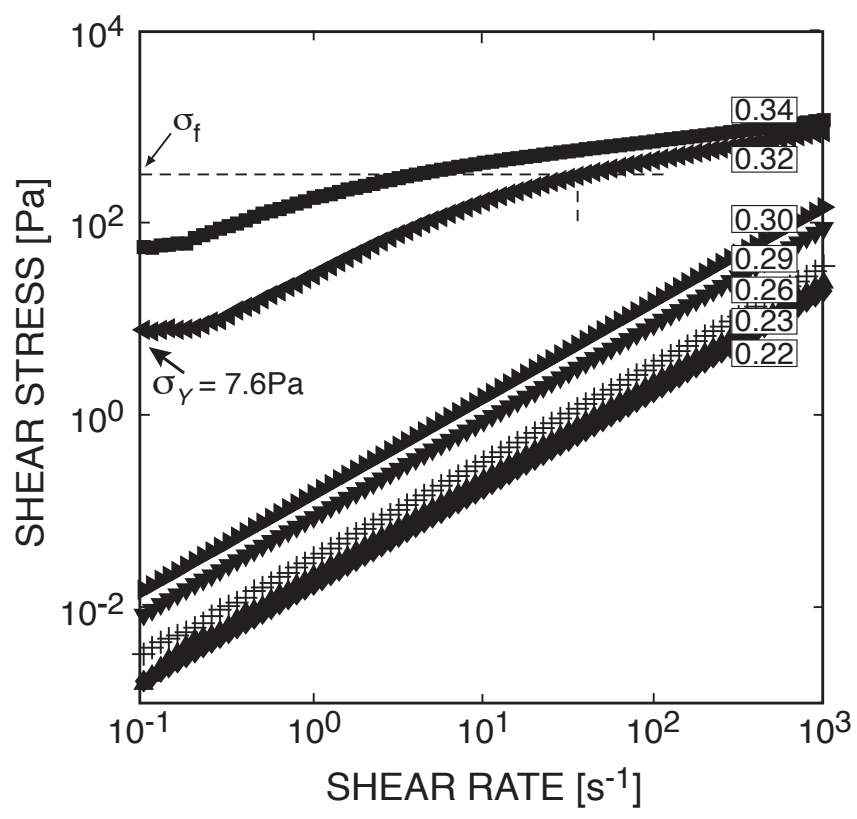

Fig. 3 Flow curve for Ludox®HS-40 samples at various $\phi_{p}$. At low shear rate, an apparent yield stress $\sigma_{Y}$ appears for $\phi_{p} \geq 0.32$. Symbols as in Fig. 2 .

formed two different tests. In the step-and-hold function (Mezger 2002), incremental steps of temperature are made between periods of constant temperature of a given time $(1200 \mathrm{~s})$, on non-dried samples of pure Ludox $(\mathrm{R}) \mathrm{HS}-$ 40. $\mathrm{T}$ is increased from $5^{\circ} \mathrm{C}$ to $45^{\circ} \mathrm{C}$, or decreased from $45^{\circ} \mathrm{C}$ to $5^{\circ} \mathrm{C}$, while $\dot{\gamma}$ is held constant at $1 \mathrm{~s}^{-1}$. There is an interval of $1200 \mathrm{~s}$ between each temperature increment to allow the sample and the measuring system to reach thermal equilibrium. A new sample is used for each test. Figure 4 shows that the viscosity decreases as the temperature increases, but the effect is small (less than a factor of 3 over $40^{\circ} \mathrm{C}$ ) compared to the particle fraction dependence. This temperature dependence is of the same magnitude as the temperature-dependence of water viscosity (fig.4).

For higher concentration samples $\left(\phi_{p}>0.31\right)$, a timesweep test is performed where $\gamma, \omega$ and $T$ are held constant for an interval of $2000 \mathrm{~s}$ between each change in temperature. $\gamma$ and $\omega$ are fixed at $0.5 \%$ and $1 \mathrm{~s}^{-1}$, respectively, while $T$ is varied. Similarly to the step-and-hold function, the complex viscosity decreases with increasing temperature $\left(\phi_{p}=0.31\right.$; Fig. 4$)$, although measurements were limited to $T \leq 35^{\circ} \mathrm{C}$ due to drying. Additionally, measurements at $\phi_{p}=0.40$ were not possible above $22^{\circ} \mathrm{C}$, as otherwise the sample was completely dry at the end of the measurement.

\subsection{Visco-elastic properties}

The two oscillatory tests are used to estimate the viscoelastic (VE) behavior of the samples in both sol- and gelstate. The amplitude sweep test gives us the VE range

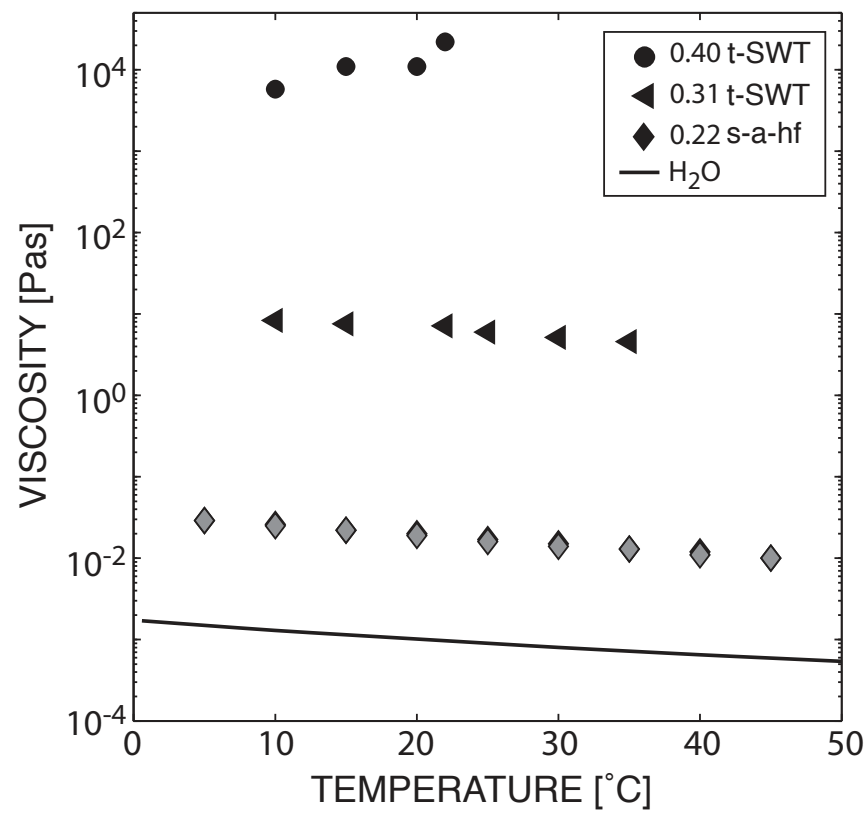

Fig. 4 Viscosity as a function of temperature. Symbols refer to different concentrations and tests. For the step-andhold function (s-a-hf), black symbols represent the descending ramp from 45 to $5^{\circ} \mathrm{C}$, and grey ones the ascending ramp, from 5 to $45^{\circ} \mathrm{C}$. The solid line shows the viscosity of pure water.

and the VE threshold, $\gamma_{L}$. During this test, the sample is subjected to an increasing oscillatory strain $\left(10^{-2}-10^{3} \%\right)$ in a logarythmic ramp profile, while frequency and temperature are kept constant $\left(\omega=10 \mathrm{~s}^{-1}, T=22^{\circ} \mathrm{C}\right)$. The measured storage and loss moduli, $G^{\prime}$ and $G^{\prime \prime}$, characterizing the stored elastic energy and the energy dissipated by heat, respectively, are plotted as a function of strain. $\gamma_{L}$ represents the largest deformation amplitude below which the measured value of $G^{\prime}$ represents the linear response of the system, i.e. the sample structure is preserved. For $\gamma>\gamma_{L}$ the system is irreversibly changed or destroyed (Ferry 1980). In this work, $\gamma_{L}$ is defined as the strain amplitude at which $G^{\prime}$ deviates by $10 \%$ from its value in the limit $\gamma \rightarrow 0$ (Sollich 1998; Metzger 2002). $\gamma_{L}$ is associated to a critical shear stress $\sigma_{L}$. We show $G^{\prime}$ and $G^{\prime \prime}$ curves exclusively for high $\phi_{p}$ (Fig. 5), at which samples exhibit non-newtonian behavior (Fig. 2). For $\phi_{p}<0.31$, the loss modulus is several orders of magnitude larger than the storage modulus and the colloidal suspensions are purely viscous (i.e. $G^{\prime}$ is not detectable in these measurements).

When $G^{\prime}$ is measurable but always smaller than $G^{\prime \prime}$, we consider that the sample is in the VE sol-state. This happens for $0.30<\phi_{p} \leq 0.34$ (Fig. 5). When $G^{\prime}$ becomes larger than $G^{\prime \prime}$, the sample is in the VE gel-state; and for $\gamma<\gamma_{L}$, the suspension is dominantly elastic (Fig. $5)$. The (linear) elastic response is limited to low strains, whereas for high strains $G^{\prime \prime}$ is always larger than $G^{\prime}$. We see that the domain of linear VE response of the samples to a given applied strain decreases with increasing 


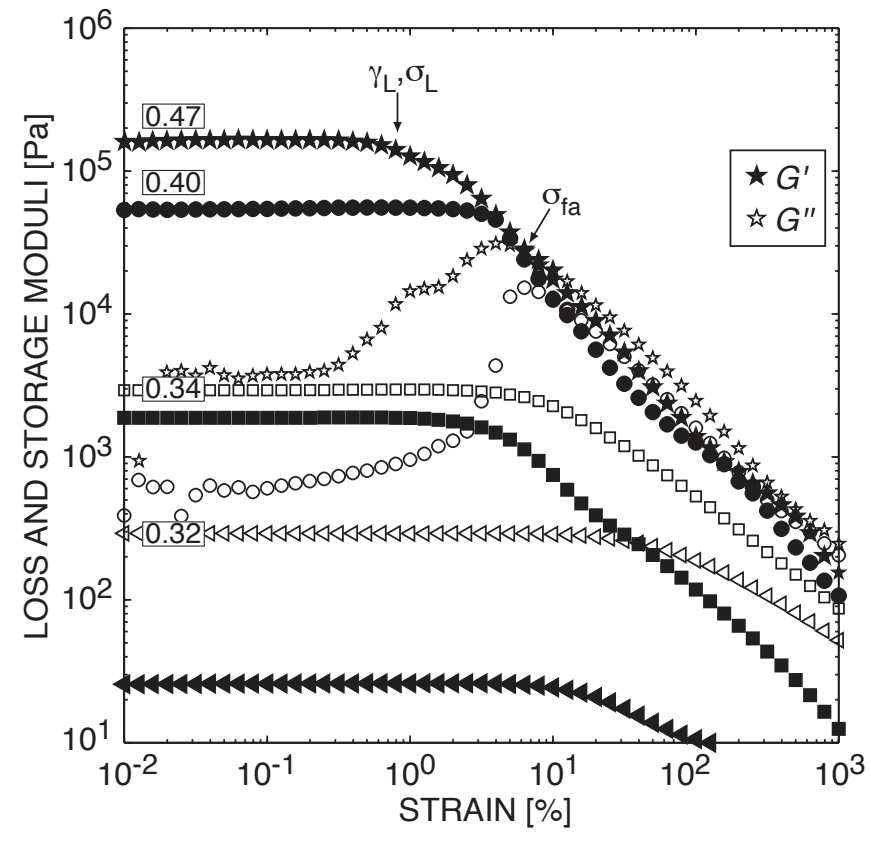

Fig. 5 Evolution of the storage and loss moduli as a function of the applied strain for $\phi_{p} \geq 0.32$. The frequency has been fixed at $10 \mathrm{~s}^{-1}$. Solid symbols refer to $G^{\prime}$, open symbols to $G^{\prime \prime}$.

particle concentration (Table 2, Fig.5). The point where the moduli intersect, i.e. $G^{\prime}=G^{\prime \prime}$ occurring for a shear stress $\sigma_{f a}$, defines the "gel point" (i.e. Drabarek et al, 2002; Di Cola et al 2009; Cao et al, 2010). For high $\phi_{p}(\geq$ $0.40), G^{\prime \prime}$ shows a peak near the gel point, which reflects the structural rearrangements occurring in the system. This is a typical feature observed in soft glassy materials (Mason and Weitz 1995; Sollich 1998; Di Cola et al 2009).

Once the limit of the linear VE range for each sample is determined, we perform a frequency sweep test on each sample in which we apply a variable oscillatory frequency, $\omega$, at a constant magnitude of oscillatory deformation. The magnitude of deformation is selected from the results of the amplitude sweep test (Fig. 5) to ensure that the test is performed in the linear VE range (i.e. $\gamma<\gamma_{L}$, Table 1). The frequency dependence of $G^{\prime}(\omega)$ and $G^{\prime \prime}(\omega)$ for different $\phi_{p}$ is shown in Fig. 6. The variation of $G^{\prime}(\omega)$ with frequency describes the structural strength of a sample (Mezger 2002). In the VE sol-state, $G^{\prime \prime}$ increases as $\omega$, whereas $G^{\prime}$ increases closer to $\omega^{2}$ at low $\omega\left(<1 \mathrm{~s}^{-1}\right)$ (table 2$)$. This behaviour is typical of hard or slightly deformable spheres suspensions (Larson, 1999). At higher concentrations in the VE gel-state, $G^{\prime}$ becomes larger than $G^{\prime \prime}$, and varies only very slowly with frequency, while $G^{\prime \prime}$ decreases with $\omega$ (Fig. 6). Values of the high-frequency elastic modulus $G_{\infty}$, obtained as the limit of $G^{\prime}$ as $\omega \rightarrow \infty$, are given in Table 2. The frequency for which $G^{\prime}=G^{\prime \prime}$ gives us the relaxation Maxwell time, $\tau_{M}=1 / \omega$, of VE samples (Fig.7). They increases from 0.003 s to $10^{3}-10^{4} \mathrm{~s}$ (Table 2 ), i.e. the larger the particle
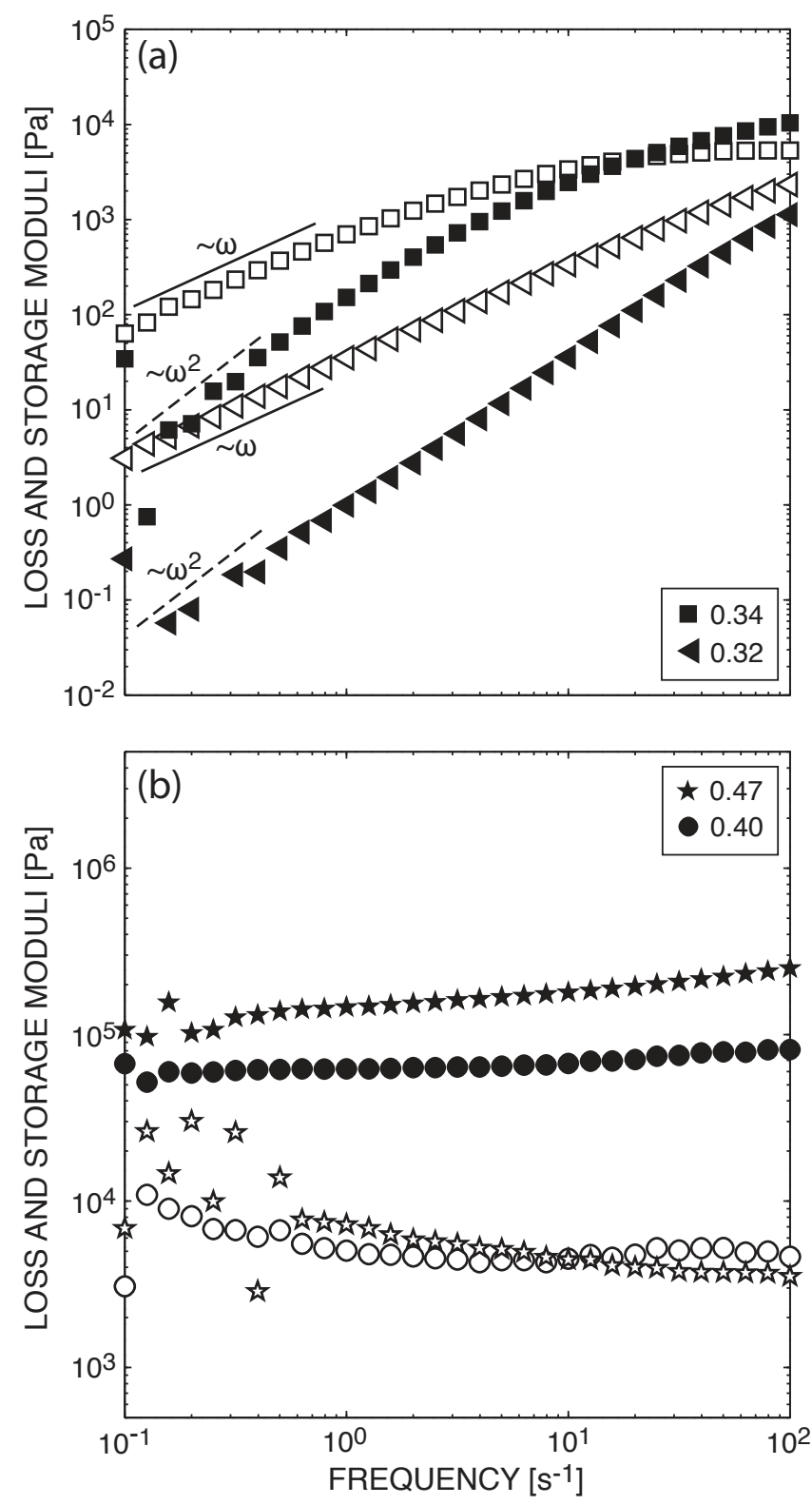

Fig. 6 Viscoelastic response determined by frequency sweep test: a) for samples with $0.32 \leq \phi_{p} \leq 0.34$; b) for samples with $0.40 \leq \phi_{p} \leq 0.47$. All measurements were performed at sufficiently low strains, $\gamma<\gamma_{L}$, to be in the linear regime (see Table 2). Solid and open symbols as in Fig. 5.

concentration, the longer the relaxation time. This is in good agreement with the values found by Mattson et al (2009) for concentrated colloidal suspensions.

\section{Measurements of the Elastic Parameters of Solid Ludox@:}

The solid samples consists in a white, translucent material which breaks in a random pattern with curvilinear edges. This constitutes evidence of a non uniform crystalline structure (probably an amorphous micro-structure, 


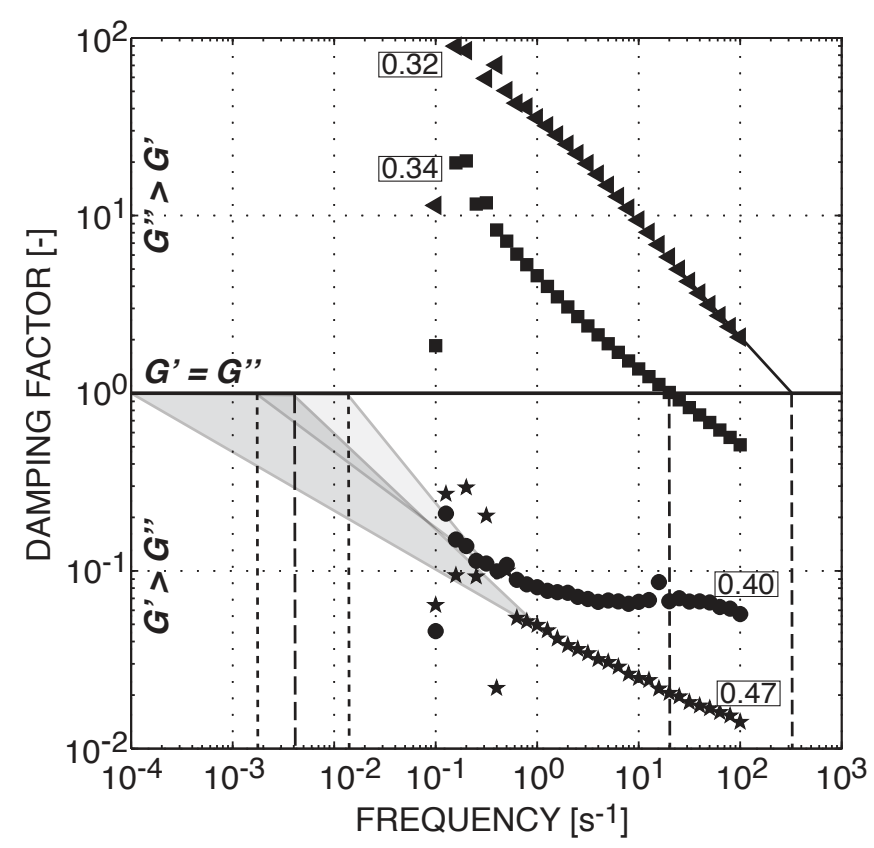

Fig. 7 Damping factor $G^{\prime \prime} / G^{\prime}$ as a function of frequency for samples with $0.32 \leq \phi_{p} \leq 0.47$. The curve intersection with the line $G^{\prime}=G^{\prime \prime}$ gives the relaxation time of the samples (see Table 2). For high $\phi_{p}$, the uncertainty is large, due to the limitations of our rheometer.

\begin{tabular}{|l|c|c|c|c|c|}
\hline No & $\begin{array}{c}\text { Pmax } \\
(\mathrm{mN})\end{array}$ & $\begin{array}{c}\text { Smax } \\
(\mathrm{mN})\end{array}$ & $\begin{array}{c}h_{\max } \\
(\mu \mathrm{m})\end{array}$ & $\begin{array}{c}E_{i} \\
\left(10^{9} \mathrm{~Pa}\right)\end{array}$ & $\begin{array}{c}\sigma_{f i} \\
\left(10^{7} \mathrm{~Pa}\right)\end{array}$ \\
\hline 1 & 500 & 172.118 & 7.137 & 2.620 & 5.79 \\
2 & 1000 & 272.718 & 13.230 & 2.590 & 4.49 \\
3 & 2000 & 390.708 & 18.880 & 2.892 & 5.43 \\
5 & 2500 & 472.451 & 25.391 & 2.703 & 4.05 \\
6 & 2750 & 463.454 & 25.293 & 2.702 & 4.43 \\
7 & 2850 & 652.623 & 25.615 & 3.621 & 4.52 \\
8 & 2950 & 658.546 & 26.488 & 3.558 & 4.42 \\
9 & 3100 & 560.689 & 27.114 & 3.043 & 4.52 \\
23 & 3200 & 603.271 & 28.299 & 3.134 & 3.75 \\
24 & 3500 & 512.459 & 30.503 & 2.573 & 3.73 \\
26 & 3600 & 629.074 & 35.340 & 2.696 & 3.28 \\
\hline
\end{tabular}

Table 3 Experiment number, maximum force applied, maximum slope of the unloading curve, contact depth, Youngs modulus and material yield stress values estimated with indentation tests.

which may contain crystalline domains). This leads to an isotropic mechanical behavior, which is fully defined by two parameters: the Young's modulus, E, and the Poisson ratio, $\nu$. We measured these parameters with two techniques. First, indentation tests give an estimate of the material yield (or failure) stress (the stress above which the deformation is irreversible), $\sigma_{f i}$, and of $E$ and $\nu$. Second, ultrasonic measurements yield an accurate measurement of $E$ and $\nu$ as long as the excitation wavelength is much greater than the typical size of the microstructure size (Ouglova et al 2006): in our case, we utilize a millimetric wavelength while the particle size is nanometric.

\subsection{Indentation tests}

Indentation tests involve pressing a diamond indenter into a material at a given force and measuring the steepest slope of the unloading curve at the instant the force is removed (Fig. 7). The unloading curve is assumed to be purely elastic immediately after unloading and to provide a direct measure of the elastic parameters of the material (Malzbender et al 2002). We perform indentation measurements using a CSM MHTX Micro-Indenter (www.csm-instruments.com) with a Vicker's style (pyramidshaped) diamond indenter tip (Poissons ratio, $\nu_{d}=0.07$, and Youngs Modulus, $\left.E_{d}=1141 \mathrm{GPa}\right)$. For our geometry, the area of indentation (i.e. the surface contact between the indenter and the material) is given by $A=$ $24.5 h_{c}^{2}+500.10^{-6} h_{c}$, where the contact depth,

$h_{c}=h_{\max }-\epsilon\left(P_{\max } / S_{\max }\right)$ depends upon the maximum force applied $P_{\max }$, a geometric factor $\epsilon(=0.74)$, and the maximum slope of the unloading curve $S_{\max }$ (i.e. Malzbender et al, 2002; note that all values are in SI units). Taking into account the non-rigid response of the indenter, measurements of $S_{\max }$ yield an estimate of the specimen Young's Modulus, $E_{i}$, of the sample through (Olivier and Pharr 1992):

$$
\frac{1}{E_{r}}=\frac{1-\nu_{d}^{2}}{E_{d}}+\frac{1-\nu^{2}}{E_{i}}
$$

where $\nu=0.18$ is the Ludox $($ Poisson ratio determined by ultrasonic measurements (see below), and the reduced Young's Modulus $E_{r}$ is given by:

$$
E_{r}=\frac{\sqrt{\pi}}{2} \cdot \frac{S_{\max }}{\sqrt{A}}
$$

Finally, a representative value of the material yield stress $\sigma_{f i}$ is estimated using the following semi-empirical relationship originally developed for metals (Tabor 1951),

$$
\sigma_{f i}=\frac{P_{\max }}{2.8 A}
$$

$\sigma_{f i}$ corresponds to the maximum stress above which the material deforms plastically, through for exemple dislocations (e.g. Schall et al, 2004). Initially zero, the applied force on each sample is increased at $200 \mathrm{mN} / \mathrm{min}$ until reaching a $P_{\max }$ between $500 \mathrm{mN}$ and $3600 \mathrm{mN}$ which is held constant for 10 minutes before unloading the sample at a rate of $P_{\max } / \mathrm{min}$. Measures of $P_{\max }$, $S_{\max }$, and $h_{\max }$ together with Eqs. (4) and (5) yield the desired values of $E$ and $\sigma_{f i}$.

Eleven separate indentation tests were performed on dry samples of Ludox@HS-40. A new sample was used each time. The values of $\sigma_{f i}$ range between $33 \mathrm{MPa}$ and $58 \mathrm{MPa}$ and the calculated values of $E_{i}$ range from 2.59 $\mathrm{GPa}$ to $3.67 \mathrm{GPa}$ (Table 2). As expected, the uncertainties on $E_{i}$ are large as the domain of linear elastic response considered to determine the slope is small (Fig. 7b). Moreover, although there is no evidence of 


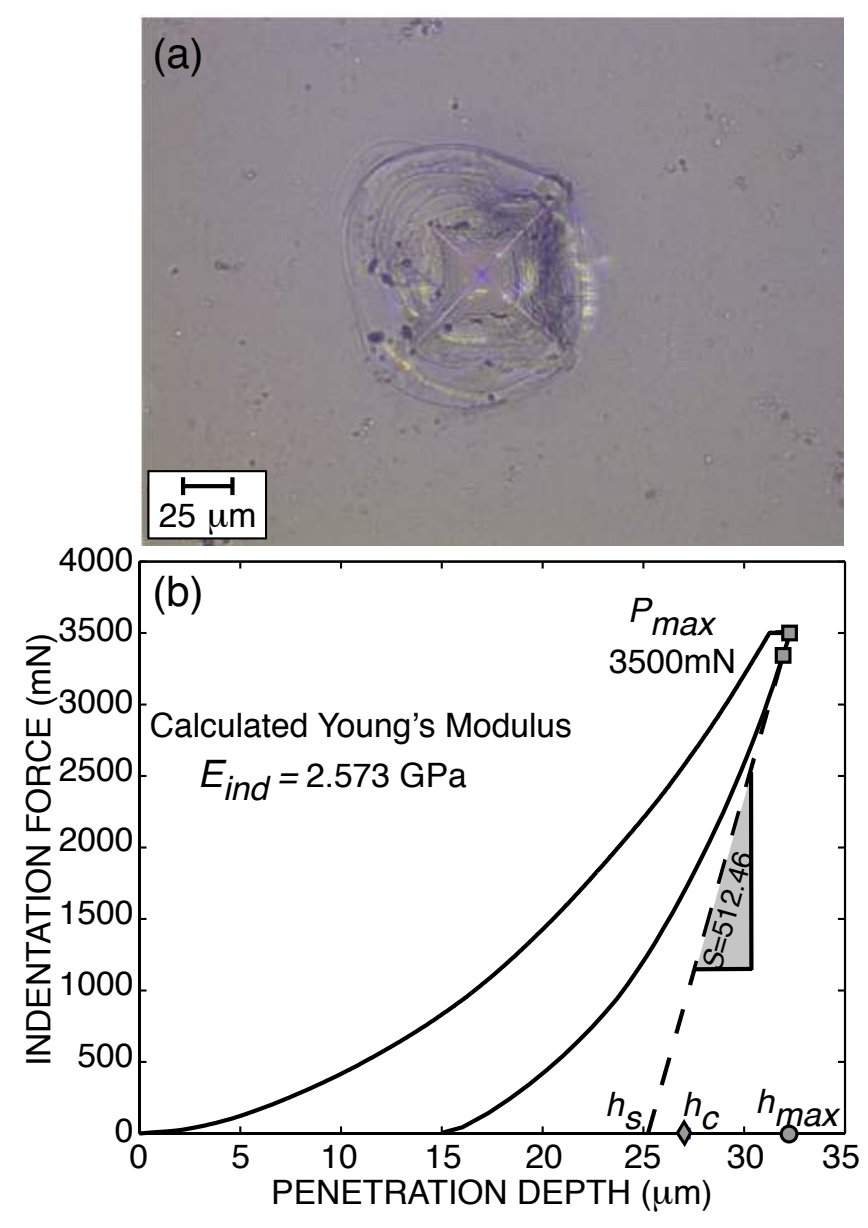

Fig. 8 Indentation test. a) samples aspect at the end of the test, b) Indentation force as a function of the penetration depth for exp.n 24 (table 3).

macroscopic fracture formation (Fig. 7a), each indentation causes permanent plastic deformation. So we expect the $E_{i}$ values to give the right order of magnitude of the Young's Modulus but to probably underestimate it.

Nonetheless, it is interesting to compare these values with published indentation data. Our $E_{i}$ values are 40 (Pauchard, 2006) to 1000 (Zarzycki, 1988) times higher than the values measured on wet gels, which is consistent with the expected decrease of $E_{i}$ with increasing water content. On the other hand, our $E_{i}$ is $40-50 \%$ smaller than the values measured by Malzbender et al (2002) on silica-filled polymeric MTMS coatings. This is probably due to the presence of the polymeric matrix which fills the gaps between the particles and hence strenghtens the material.

\subsection{Ultrasonic measurements}

The ultrasonic measurements were used to determine the Youngs Modulus and Poissons ratio of the solid Ludox $\mathbb{R} \mathrm{HS}$ 40. Panametrics V109 (pressure waves) and V154 (shear waves) transducer pairs were placed on either side of a dried sample and coupled to the sample using honey. We refer to the Youngs Modulus measured with this technique, as $E_{u s}$. The central frequencies of the transducers are 5.0 MHz and 2.5 MHz for the V109 and V154 respectively. The travel-times of the first arrival and subsequent echoes are measured on an oscilloscope. In order to avoid the uncertainty on the time origin, we consider only the flight times measured between one, two or three echoes at the receiver tranducer in the calculation of wave speeds (Table 3 ). Three P-wave arrivals and two S-wave arrivals are measured. Using the calculated wave speeds, $E_{u s}$ and $\nu$ are determined by (Royer and Dieulesaint, 1989):

$$
E_{u s}=\rho_{\text {dried }} V_{s}^{2} \frac{3 V_{p}^{2}-4 V_{s}^{2}}{V_{p}^{2}-V_{s}^{2}}
$$

and

$$
\nu=\frac{V_{p}^{2}-2 V_{s}^{2}}{2\left(V_{p}^{2}-V_{s}^{2}\right)}
$$

The measured sound velocities are $3218 \mathrm{~m} / \mathrm{s} \pm 0.2 \%$ for pressure waves and $2018 \mathrm{~m} / \mathrm{s} \pm 3 \%$ for shear waves (Table $3)$. Thus, Eqs. (6) and (7) yield $E_{u s}=16.1 \pm 0.6 \mathrm{GPa}$ and $\nu=0.18 \pm 0.03$.

The Young's Modulus, and consequently the elastic modulus, estimated by indentation is about five times lower than that measured with the ultrasonic technique (Fig. 11). This can be due both to the limitations of the indentation technique, and to the different space and time scales on which the tests are performed. Indeed, even though we do not see macroscopic fractures on Fig. 7a, the material appears strongly deformed and damaged: most probably micro-cracking exists under the indenter, which will weaken the elasticity of the material. Moreover, with indentation, a thin layer of the material of $<35.34 \mu \mathrm{m}$ of thickness undergoes deformation at a strain rate of $\sim 3.0 \times 10^{-3} \mathrm{~s}^{-1}$. With the ultrasonic method, the waves examine the whole thickness of the sample (typically $2.25 \mathrm{~mm}$ ) at a strain rate of $\sim 1 \mathrm{~s}^{-1}$ (when assuming a nanometric amplitude of the ultrasonic wave, e.g. Royer and Dieulesaint 1989). The sample has thus less time to deform plastically in the ultrasonic case, ensuring an optimal elastic response.

\section{Discussion}

\subsection{Newtonian viscosity: aggregation and/or electrostatic effects?}

Even for our lower range of $\phi_{p}\left(0.22 \leq \phi_{p} \leq 0.30\right)$ where the viscosity remains newtonian, the average separation distance between particles is smaller than the particle size (Madeline et al, 2007), so that we always are dealing with dense suspensions (e.g. Stickel and Powell, 2005). It is therefore expected that the models developed for 


\begin{tabular}{|l|c|c|c|c|c|}
\hline P-waves & & & S-waves & & \\
\hline$i \rightarrow j$ & $\begin{array}{c}\Delta t_{i j} \\
\left(10^{-6} \mathrm{~s}\right)\end{array}$ & $\begin{array}{c}V_{P}^{i} \\
\left(10^{3} \mathrm{~m} / \mathrm{s}\right)\end{array}$ & $i \rightarrow j$ & $\begin{array}{c}\Delta t_{i j} \\
\left(10^{-6} \mathrm{~s}\right)\end{array}$ & $\begin{array}{c}V_{S}^{i} \\
\left(10^{3} \mathrm{~m} / \mathrm{s}\right)\end{array}$ \\
\hline $0 \rightarrow 1$ & 0.88 & 2.550 & $0 \rightarrow 1$ & 1.29 & 1.744 \\
$1 \rightarrow 2$ & 1.40 & 3.226 & $1 \rightarrow 2$ & 2.30 & 1.957 \\
$1 \rightarrow 3$ & 2.79 & 3.214 & $1 \rightarrow 3$ & 4.33 & 2.079 \\
$1 \rightarrow 4$ & 4.20 & 3.214 & & & \\
\hline
\end{tabular}

Table 4 Travel times of the first arrival and subsequent multiples and resulting P- and S- waves speed calculated for the ultrasound measurements. Only the multiples have been considered in the calculation of the elastic parameters (see text).

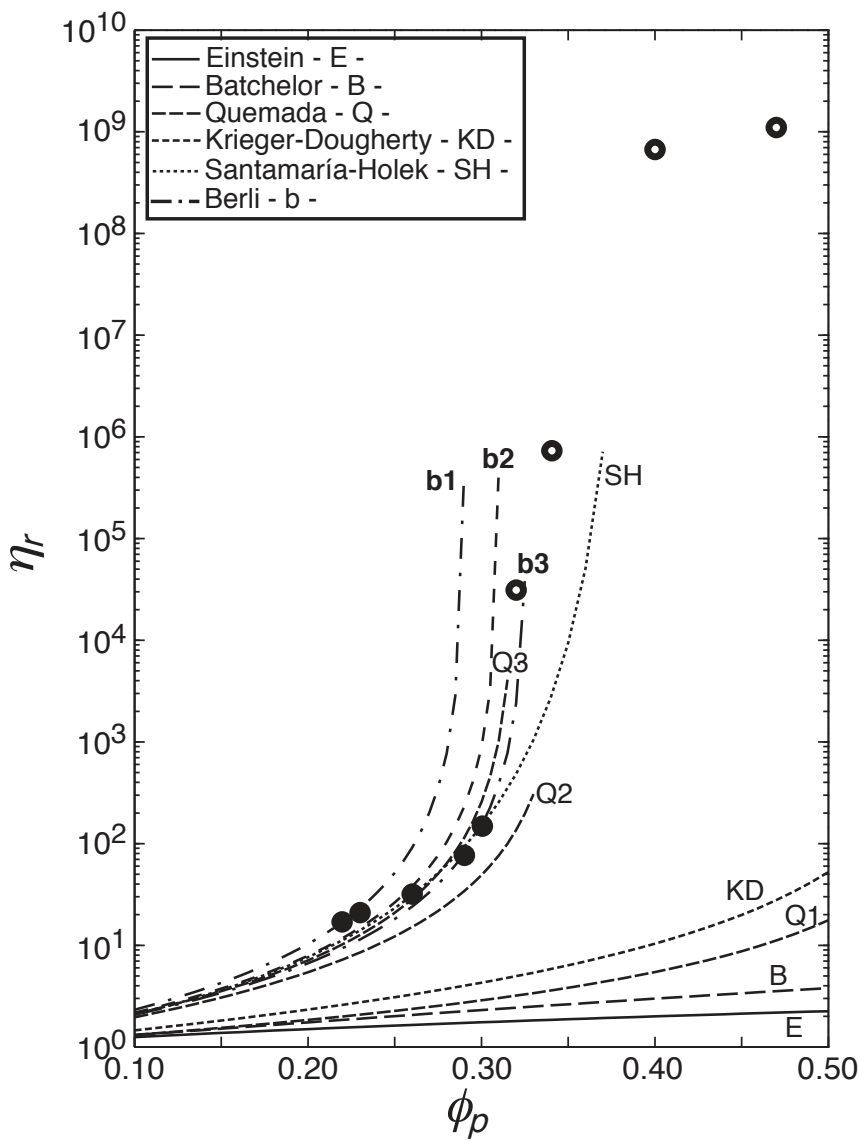

Fig. 9 Relative viscosity $\eta_{r}=\eta / \eta_{s}$ as a function of particle volume fraction. Empty symbols describe the experimental data with $0.22 \leq \phi_{p} \leq 0.30$; filled symbols, the samples with $0.32 \leq \phi_{p} \leq 0.47$. Lines refer to theoretical models, as showed by the legend. The curves from Quemada (1977) have been calculated for $\phi_{c}=0.58$ (curve Q1), $\phi_{c}=0.35$ (curve Q2) and $\phi_{c}=0.31$ (curve Q3). Krieger-Dougherty (1959) model has been calculated by imposing $[\eta]=2.5$ and $\phi_{m}=0.58$. The curve from Santamaría-Holek and Mendoza (2010) that best fits the experimental data is given by $[\eta]=5.86$ and $\phi_{c}$ $=0.386$. Curves $\mathrm{a}, \mathrm{b}, \mathrm{c}$ were obtained following the effective particle radius approach of Berli et al (2005): curve a for $r_{e f f}=9.05$; curve $\mathbf{b}$ for $r_{e f f}=8.80$; curve $\mathbf{c}$ for $r_{e f f}=$ 8.70 (equation 12 with $\phi_{c}=0.51$, the value measured in our solid samples. Taking $\phi_{c}=0.58$ at the glass transition would increases the calculated radii by $4 \%$ )). the dilute limit (Einstein, 1906), even when they include two-particles hydrodynamic interactions (Batchelor, 1970), fail to predict the measured viscosity (fig.9). The increase of viscosity is also much larger than that predicted by the semi-empirical relationship obtained by Krieger and Dougherty (1959) for colloidal solutions with $\phi_{p} \geq 0.1$, where they incorporate the excluded volume (Fig.9):

$$
\eta=\eta_{s}\left(1-\frac{\phi_{p}}{\phi_{m}}\right)^{-[\eta] \cdot \phi_{m}}
$$

where $\eta_{s}$ is the viscosity of water. $[\eta]$ is the intrinsic viscosity, and for spherical particles $[\eta]=2.5 \phi_{m}$ is the particle fraction at which the solution becomes solid and is usually taken at the glass transition for hard spheres: $\phi_{m}=0.58$. If we take instead $\phi_{m}=0.51$, the particle volume fraction measured in our solid samples, it does not fit better the data for the range considered. The latest version of this model includes steric exclusion as well as the hydrodynamic interactions of multiple particles (Santamaría-Holek and Mendoza, 2010) :

$$
\eta=\eta_{s}\left(1+\frac{\phi_{p} \cdot \phi_{c}}{\phi_{c}-\phi_{p}+\phi_{p} \cdot \phi_{c}}\right)^{-[\eta]}
$$

where $\phi_{c}$ is a critical volume fraction and $[\eta]$ is the low filling fraction intrinsic viscosity, which value depends on the particle shape. The best fit of our data implies $[\eta]=5.86$ and $\phi_{c}=0.386$. However, fig. 9 shows that the divergence of viscosity at high particle content is less abrupt than observed, and the viscosity value at $22 \%$ is too low compared to real data. Besides, the value of $[\eta]$ would require very elongated particle shapes (i.e. Santamaría-Holek and Mendoza, 2010), which is not observed (Madeline et al, 2007).

A different approach to calculate the viscosity of suspensions is based on the divergence of viscosity expected theoretically near the percolation threshold (Quemada, 1977):

$$
\eta=\eta_{s}\left(1-\frac{\phi_{p}}{\phi_{c}}\right)^{-2}
$$

where now $\phi_{c}$ is the particle volume fraction above which the fluid has solid properties. We see on Fig.9 that a $\phi_{c}$ taken at the glass transition (i.e. $\phi_{c}=0.58$, curve Q1) does not fit the data at all. However, decreasing $\phi_{c}$ towards 0.31 , the limit above which our samples show a 
yield stress and non-newtonian behaviour, gives a much better agreement (curves Q2 and Q3 on Fig.9). This decrease can be due to two phenomena: the formation of particle aggregates, or the presence of an electrical double-layer.

Particle aggregates: It is now well documented that gelation of dilute colloids suspensions occurs through the growth of particles clusters (e.g. Shih et al, 1989, Lin et al, 1989). The value of $\phi_{c}$ is then related to the amount of fluid entrapped within each aggregate (e.g. Tsenoglou, 1990; Aubry et al, 1998; Chougnet et al, 2007; Bullard et al, 2009). The structure of the colloidal aggregates can be described by a fractal behaviour with a fractal dimension $D$ ranging between 1.6 and 2.2 (e.g. Shih et al, 1989; Lin et al, 1989; Wang et al 2003; Prasher et al 2006; Cao et al, 2010). The equation of Krieger and Dougherty (eqn.8) can then be rewritten as (Chen et al, 2007):

$$
\eta=\eta_{s}\left(1-\frac{\phi_{p}}{\phi_{m}} \cdot\left(\frac{a_{e f f}}{a}\right)^{3-D}\right)^{-[\eta] \cdot \phi_{m}}
$$

where $a_{e f f}$ is the effective size of the fractal aggregate. Inversely, $a_{\text {eff }}$ can be deduced from eqn.(11) and the viscosity of the suspension. In our case, it would give cluster sizes ranging from 4 times to twice the particle size, and decreasing with increasing particle volume fraction. The small size of the cluster makes the hypothesis of a fractal behaviour questionable. Besides, we would expect an increase of the cluster size, rather than a decrease, as gelation proceeds. So predictions for aggregates do not explain our data well. The absence of clusters for the low particle fractions is in agreement with the SANS experiments of Madeline et al (2007) which show a regular network of repelling isolated silica particles for $\phi_{p}=0.22$.

Electrical double-layer (EDL): the high density of $\mathrm{SiO}^{-}$groups covering the silica surfaces, and the layer of $\mathrm{Na}^{+}$counterions in the aqueous phase which surrounds each particle, form the EDL, producing strong interparticle repulsions (Fig.12). The EDL presence therefore increases the particle radius (e.g. Allain et al, 1993). In the case of overlapping electronic clouds, soft effective particles are probably a better image than hard spheres. However, models to predict soft spheres viscosity are still lacking (Quemada and Berli, 2002; Russel, 2009), in part because electro-viscous effects are still not completely described (e.g. Sherwood et al, 2000; Russel, 2009). But a model for effective hard spheres can already gives us useful insight in our dispersions behaviour. Following Allain et al (1993) and Berli et al (2005), Quemada's model can be written for effective particles of radius $r_{\text {eff }}$ :

$$
\eta=\eta_{s}\left(1-\frac{\phi_{p}}{\phi_{c}}\left(\frac{r_{e f f}}{a / 2}\right)^{3}\right)^{-2}
$$

where we take $\phi_{c}=0.51$ (i.e. the particle volume fraction of our solid samples). So from the evolution of the

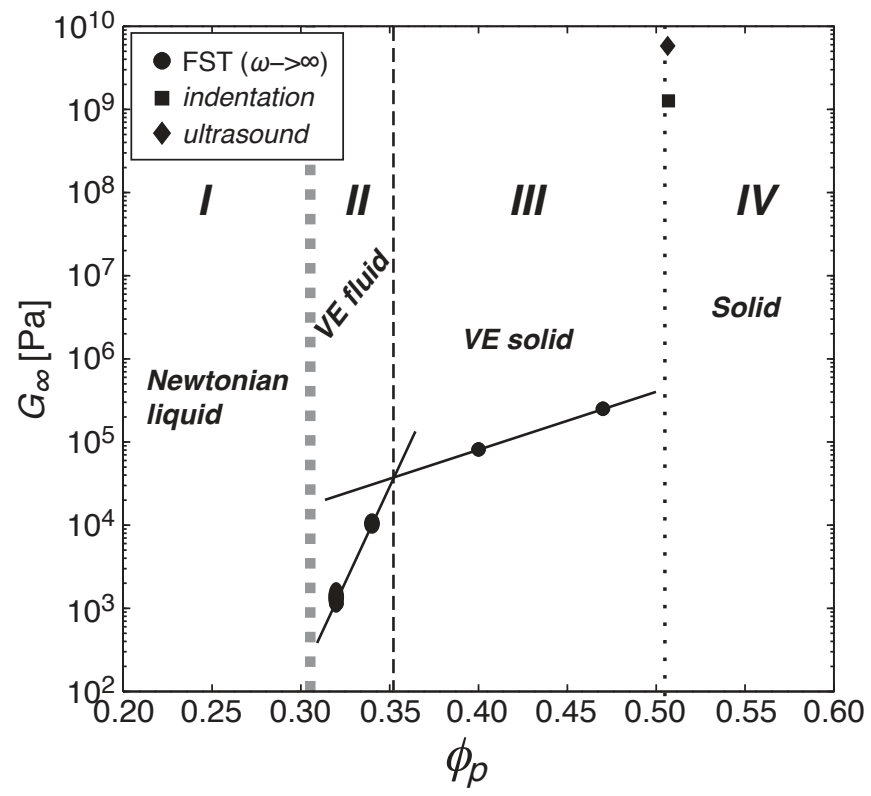

Fig. 10 High frequency elastic modulus, $G_{\infty}$, as a function of particle volume fraction. Symbols indicate the methods employed. The symbol size represents the measurement uncertainty. For the solid samples, $G_{\infty}$ is calculated from the Young's Modulus through the relationship $G_{\infty}=E / 2(1+\nu)$ (Mezger, 2002). Four different rheological regimes can be identified as $\phi_{p}$ increases.

viscosity, we can deduce the evolution of the effective radius of the particles. Table 1 shows that the predicted radii (around $9 \mathrm{~nm}$ instead of the $7.5 \mathrm{~nm}$ of the silica particles) are compatible with the average interparticle distance (19 nm) evaluated by Madeline et al (2007). Besides, the viscosity data (Fig.9) can be explained if the effective radius decreases as $\phi_{p}$ increases from 0.22 to 0.29 and then levels off : this means that the EDL is progressively squeezed down to a certain limit.

In conclusion, the increased viscosity values in the newtonian regime are well explained if we consider the presence of the EDL around individual silica particles, and we do not need to invoke the formation of aggregates.

\subsection{Four stages from liquid to solid}

The evolutions of the high frequency elastic modulus and of the yield stresses as the particle content increases are summarized on Fig.10 and 11, respectively. We can distinguish four stages. Moreover, $G_{\infty}$ and $\sigma_{f}$ are both related to the shape of the particle interaction potential created by the attractive van der Waals forces and repulsive electrostatic interactions (Fig.12), in particular through the relationship (e.g. Larson, 1999):

$$
G_{\infty} / \sigma_{f}=2 a / D_{0}
$$


where $D_{0}$ is the gap between particle surfaces (Table 2). So we can discuss the different regimes in terms of the structure of the colloidal suspensions.

Regime $\mathrm{I}$ is the newtonian regime that we discussed in the previous section, where the suspensions are composed of a network of individual silica particles, each surrounded by an EDL. This regime ends when the EDL cannot be squeezed anymore.

Looking only at the boundary of the linear VE regime (e.g. $\sigma_{f}$ on Fig.11), regimes II and III would be counfonded. However, $G_{\infty}$ (Fig.10) and $D_{0}$ (Table 2) data tell a different story.

Regime II is characterized by the appearance of solid properties, such as elasticity (Fig.5 and Fig.6), and a yield stress $\sigma_{Y}$ under which the suspension does not flow (Fig.2 and 3). That means that the suspension begins to have a solid squeleton which can resist small deformations. At the transition between I and II, the solutions become cloudy, pointing to the formation of aggregates. A similar phenomenon was observed by Trompette and Clifton (2003) and Madeline et al (2007) during the gelation and the growth of particles aggregates of Ludox $(\mathrm{R} H \mathrm{H} 40$ at low volume fraction and low $\mathrm{pH}$. The high frequency elastic modulus $G_{\infty}$ then increases with the connectivity of the solution and the growth of aggregates (e.g. Shih et al, 1990; Grant and Russel, 1993; Channel and Zukoski, 1997; Cao et al, 2010). For gelation by growth of fractal flocs at low particle fractions, $G_{\infty} \sim \phi_{p}^{y}$ with $3 \leq y \leq 5$ ( Krall and Weitz, 1997; Channel and Zukoski, 1997). Here, the increase is much steeper, with $y \sim 30$. Hence, we probably have to find a different mechanism to explain the data. We propose that regime II is due to the peculiar behaviour of the EDL: the increasing volume fraction of particles tend to squeeze the EDL, which becomes more and more difficult because of the repulsive forces. So at one point, it it is energetically more favourable to remove in some places the EDL and constitute aggregates of particles while leaving the EDL intact around the other particles, than to reduce uniformly the thickness of the EDL throughout the whole suspension. This comes from the shape of the interparticle potential, which show two stable points ("p" and "s" of Fig.12) and an intermediate unstable one. Materials composed by such a collection of finite or infinite non linear springs (Ericksen,1975; Puglisi and Truskinovsky, 2005) develop instabilities under stress and show strain localization. Such behavior is met, at the macroscale, for example in foams (Del Piero and Pampolini, 2010). Here, one may think that the colloid, for 0.32 $\leq \phi_{p} \leq 0.34$, consists of a mixture of two phases: one of them with squeezed EDL (say around point "s") and the second with already joined EDL's and much closer particles (say around point "p"). The mean gap between the particles $D_{0}$ decreases very steeply between $\phi_{p}=$ 0.32 , where $D_{0}$ is close to the values $\sim 4 \mathrm{~nm}$ found in the newtonian regime, to $\phi_{p}=0.34$ (Fig.12), where $D_{0}$ approaches the values found in the pasty samples. This

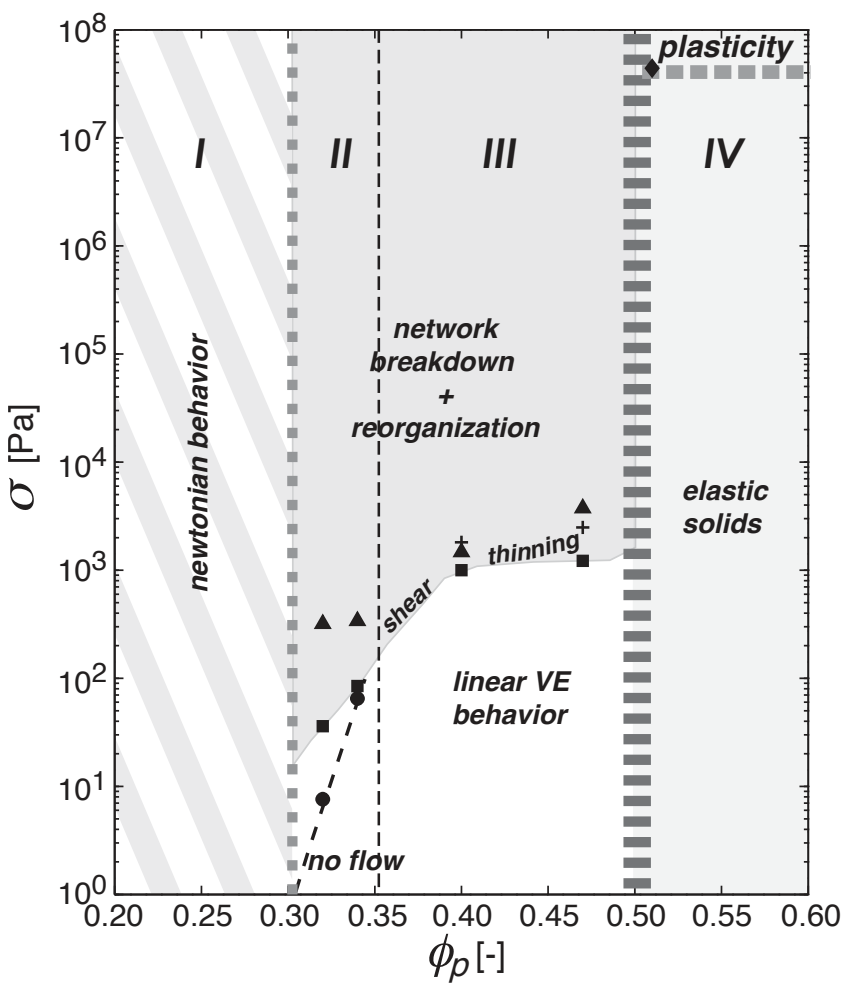

Fig. 11 Regime diagram as a function of the applied stress and the particle volume fraction. Symbols indicate the different yield stresses evaluated through the different tests. Circles refer to $\sigma_{Y}$, squares to $\sigma_{L}$, triangles to $\sigma_{f}$ and crosses to $\sigma_{f a}$, measured by flow test and amplitude sweep test, respectively. Grey scales identify the different regimes.

sharp decrease would reflect the increasing importance of aggregates in the suspension.

In Regime III , most of the particles have aggregated (i.e. have fallen into the potential sink "p" of Fig.12). However, they can still rotate and rearrange themselves, especially under high shear, where shear banding and reorganization of the particles in planes gliding one on the other prevail (e.g. Ackerson and Pusey, 1988; Drabarek et al, 2000). The organization of the particles is coherent with the observation that the samples (for $\phi_{p}=0.40$ and 0.47 ) become transparent again. This pasty state has already been reported in directional drying of colloidal solutions (e.g. Dufresne et al, 2003; Goehring et al, 2009). The elastic modulus $G_{\infty}$ continues to increase, but much slower that in the previous regime, with $y \sim 6.9$. We may interpret this growth as the aggregation of flocs.

Then, for $\phi_{p} \sim 0.51$, we have the classical colloidal hard sphere transition, leading to the solid state (Regime IV) where the particles are trapped within the cages constituted by their neighbours: the structure is at arrest. Given $G_{\infty}$ and $\sigma_{f i}, D_{0}$ is very small, $\sim 2.10^{-10} \mathrm{~m}$, and the particles are clumped together into a single mass. Irreversible deformation caused by plasticity or fracture will occur only for much higher stresses (fig.11). 


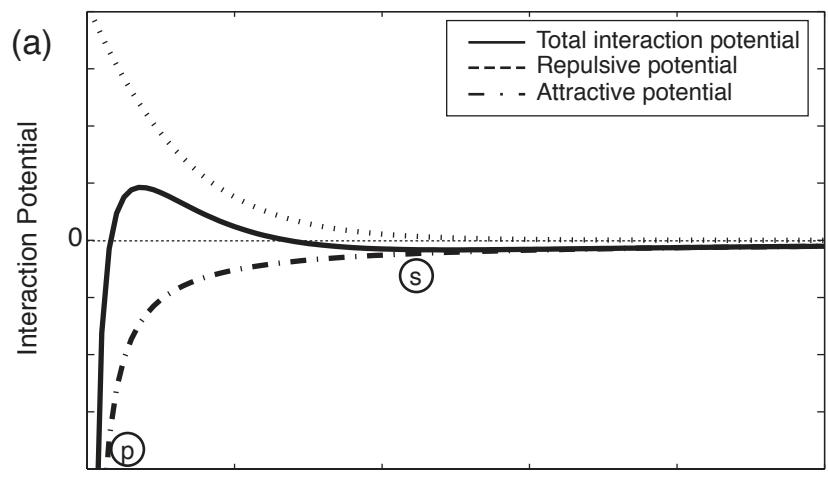

Distance from particle surface

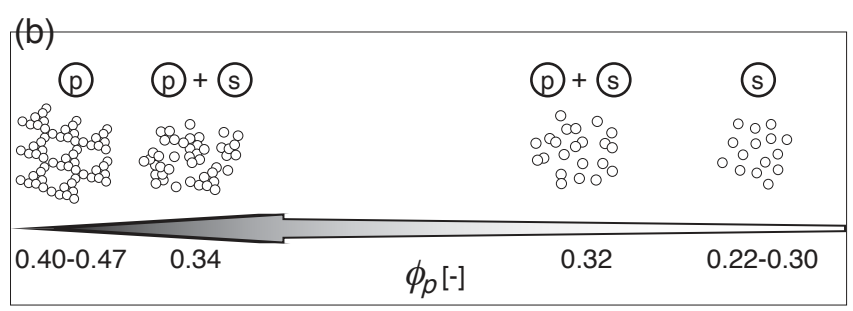

Fig. 12 a) Particle interaction potential as a function of the distance away from the surface of a particle, according to DLVO theory. "p" and "s" show the two stable positions (see text). b) Cartoon showing the samples behaviour and the formation of the aggregates as a function of particle separation.

\section{Conclusions}

We have investigated the rheological and the mechanical behavior of hard-sphere silica colloidal suspensions (Ludox®HS-40) as a function of particle concentration and temperature. Four regimes are identified with increasing particle content; they are related to the existence and behaviour of the electrical double layer (EDL) around each particle. Semi-diluted suspensions $(0.22 \leq$ $\phi_{p} \leq 0.30$ ), which do not flocculate due to the EDL, show Newtonian behavior (I). At higher particle contents, the suspensions exhibit two viscoelastic (VE) shear thinning regimes : semi-concentrated fluids (II, $0.31 \leq \phi_{p} \leq 0.35$ ) in which particles form aggregates of quickly increasing size, and concentrated strain sensitive samples with paste-like aspect and high elastic modulus (III, $0.35 \leq$ $\left.\phi_{p} \leq 0.47\right)$. Regime II can be explained by a sudden partial collapse of the EDL as it is progressively squeezed with increasing $\phi_{p}$. At higher particle concentration, the suspensions undergo a glass transition and behave as an elastic-brittle solid (IV, $\phi_{p}=0.51$ ).

Acknowledgements: This work benefited from discussions with Ludovic Pauchard, Véronique Lazarus, Georges Gauthier, Mourad Chekchaki, Fabien Mahaut, Neil Ribe and John Sherwood. E. Di Giuseppe was funded by a joint fellowship from the Swiss National Science Foundation (Subside nr. PBEXP2-122908) and the French Agence Nationale de la Recherche (PTECTO NT09-604042). Eric Mittelstaedt was funded by an International Fel- lowship from National Science Foundation (USA). The rheometer was supported by the Triangle de la Physique, and the indenter by the French Agence Nationale de la Recherche ("Morphologies" JCJC-05-0029).

\section{References}

1. Ackerson BJ, Pusey PN (1988) Shear-induced order in suspensions of hard-spheres., Phys Rev Lett 61:1033-1036.

2. Aubry T, Largenton B, Moan M (1998) Study of aggregate density in fumed silica suspensions. J Colloid Interface Sci 202:551553.

3. Batchelor G (1970) The stress system in a sus- pension of force-free particles. J. Fluid Mech. 41:54570.

4. Berli CLA, Deiber JA, Quemada D (2005) On the viscosity of concentrated suspensions of charged colloids. Latin American Applied Research, 35:15-22.

5. Birdi KS (1997) Handbook of Surface and Colloid Chemistry CRC Press Inc.

6. Bullard JW, Pauli AT, Garboczi EJ, Martys NS (2009) A comparison of viscosity-concentration relation- ships for emulsions. J Colloid Interface Sci 330:186-193.

7. Buscall R, McGowan IJ, Mills PDA, Stewart RF, Sutton D, White LR, Yates GE (1987) The rheology of strongly flocculated suspensions. J. Non-Newtonian Fluid Mech 24:183-202.

8. Cao XJ, Cummins HZ, Morris JF (2010) Structural and rheological evolution of silica nanoparticle gels. Soft Matter. DOI: 10.1039/c0sm00433b.

9. Channel GM, Zukoski CF (1997) Shear and compressive rheology of aggregated alumina suspensions. AiCHE J. 43:1700-1708 .

10. Chen H, Ding Y, Tan C (2007) Rheological behaviour of nanofluids. New J Phys 9:367.

11. Chevalier J, Tillement O, Ayela F (2009) Structure and rheology of $\mathrm{SiO} 2$ nanoparticle suspensions under very high shear rate. Phys Rev E 80:051403.

12. Chougnet A, Audibert A, Moan M (2007) Linear and non-linear rheological behaviour of cement and silica suspensions. Effect of polymer addition. Rheol Acta 46:793:802.

13. Dames B, Morrison BR, Willenbacher N (2001) An empirical model predicting the viscosity of highly concentrated, bimodal dispersions with colloidal interactions. Rheol Acta 40:434-440.

14. Del Piero G, Pampolini G (2010) On the rate-dependent properties of open-cell polyurethane foams. Technische Mechchanik 30:74:84.

15. Dickinson E (1992) An introduction to Food Colloids Oxford Univ Press . New York

16. Di Cola E, Moussad A, Sztucki M, Narayanan T, Zaccarelli E (2009) Correlation between structure and rheology of a model colloidal glass. J Chem Phys 131:144903.

17. Di Giuseppe E, Davaille A (2010) Initiation of subduction in the laboratory from solutal convection in a complexrheology fluid. Geophys Res Abstract 23:07954.

18. Drabarek E, Bartlett JR, Hanley HJM, Woolfrey JL, Muzny CD, Butler BD (2000) Shear-Induced Restructuring of Colloidal Silica Gels. J. Sol-Gel Sci Tech 19:279283 
19. Drabarek E, Bartlett JR, Hanley HJM, Woolfrey JL, Muzny CD (2002) Effects of Processing Variables on the Structural Evolution of Silica Gels. Inter J Thermophys 23:145-159.

20. Dufresne ER, Corwin EI, Greenblatt NA, Ashmore J, Wang DY, Dinsmore AD, Cheng JX, Xie XS, Hutchinson JW, Weitz DA (2003) Flow and Fracture in Drying Nanoparticle Suspensions. Phys Rev Lett 91:224501.

21. Einstein A (1906) Eine neue bestimmung der molekuldimension. Ann Phys. 28:207-227.

22. Ericksen JL (1975) Equilibrium of bars. J. Elasticity. 5:191201.

23. Ferry JD (1980) Viscoelastic Properties of Polymers John Wiley and Sons Inc (1980) New York

24. Gauthier G, Lazarus V, Pauchard L (2007) Alternating crack propagation during directional drying. Langmuir 23: 4715-4718. doi: 10.1021/la063702w.

25. Gauthier G, Lazarus V, Pauchard L (2010) Shrinkage star-shaped cracks: Explaining the transition from 90 degrees to 120 degrees. Europhys Lett 89:10.1209/0295$5075 / 89 / 26002$.

26. Grant MC, Russel WB (1993) Volume-fraction dependence of elastic moduli and transition temperatures for colloidal silica gels. Phys Rev E 47:2606-2614.

27. Jeffrey DJ, Acrivos A (1976) The rheological properties of suspensions of rigid particles. AIChE J. 22:417-432.

28. Jones DQR, Leary B, Boger DV (1991) The Rheology of a Concentrated Colloidal Suspension of Hard Sphere. J Colloid Interface Sci 147:479-495.

29. Krall AH, Weitz DA (1998) Internal dynamics and elasticity of fractal colloidal gels. Phys. Rev. Lett. 80:778781.

30. Krieger IM, Dougherty TJ (1959) A mechanism for nonnewtonian flow in suspensions of rigid spheres. Trans Soc Rheology 3:137-152.

31. Larson RG (1999) The structure and rheology of complex fluids Oxford University Press. New York

32. Lin MY, Lindsay HM, Weitz DA, Ball RC, Klein R, Meakin (1989) P. Universality of colloid aggregation. Nature 339:360-362.

33. Madeline JB, Meireles M, Bourgerette C, Botet R, Schweins R, Cabane B (2007) Restructuring of colloidal Cakes during Dewatering. Langmuir 23:1645-1658.

34. Malzbender J, Den Toonder JMJ, Balkenende AR, de With G (2002) Measuring mechanical properties of coatings: a methodology applied too nano-particle-filled sol-gel coatings on glass. Mater Sci Engin R 36:47-103.

35. Mason TG, Weitz DA (1995) Linear Viscoelasticity of Colloidal Hard Sphere Suspensions near the Glass Transition. Phys Rev Lett 75:2770-2773.

36. Mattsson J, Wyss HM, Fernadez-Nieves A, Miyazaki K, $\mathrm{Hu}$ Z, Reichman DR, Weitz DA (2009) Soft colloids make strong glasses. Nature 462:83-86.

37. Mezger TG (2002) The Rheology Handbook: for Users of Rotational and Oscillatory Rheometers. Hannover, Germany

38. Olivier OC, Pharr GM (1992) An improved technique for determining hardness and elastic-modulus using load and displacement sensing indentation experiments. J Mater Res 7:1564-583.

39. Ouglova A, Berthaud Y, Franois M and Foct F (2006) Mechanical properties of an iron oxide formed by corrosion in reinforced concrete structures, Corrosion Science 48:3988-4000.
40. Park J, Park SB, Yang SM, Hong WH, Choi CR, Kim JH (2005) Rheological Characterization and optimization of gelled electrolyte for sealed lead-acid batteries by Small Amplitude Dynamic Oscillation Measurement. J Non-Crystalline Solids 351:2352-2357.

41. Pauchard L, Parisse F, Allain C (1999) Influence of salt content on crack patterns formed through colloidal suspension desiccation. Phys Rev E 59:3737-3740.

42. Pauchard L, Patterns caused by buckle-driven delamination in desiccated colloidal gels (2006) Europhys Lett 74:188-194.

43. Puglisi G, Truskinovsky L (2005) Thermodynamics of rate independent plasticity. J Mech Phys Sol, 53:655-679.

44. Prasher R, Evans W, Meakin P, Fish J, Phelan P, Keblinski P (2006) Effect of aggregation on thermal conduction in colloidal nanofluids. Appl Phys Lett 89: 143119.

45. Quemada D (1977) Rheology of concentrated disperse systems and minimum energy-dissipation principle 0.1 . Viscosity-concentration relation- ship. Rheol Acta 16, 8294.

46. Quemada D, Berli C (2002) Energy of interaction in colloids and its implications in rheological modeling. Advances in Colloid and Interface Science 98:51-85.

47. Roscoe R (1952) The viscosity of suspensions ofrigid spheres. Br J Appl Phys 3:267-269

48. Royer D, Dieulesaint E (1989) Optical measurements of displacements of amplitude 10-4 to 10-2 anfstrom Application to elastic-waves. Revue Phys Appl 24:833-846.

49. Rueb CJ, Zukoski CF (1997) Viscoelastic properties of colloidal gels. J Rheol 41:197217.

50. Russel WB (1978) The rheology of suspensions of charged rigid spheres. J Fluid Mech. 85:209232.

51. Russel WB (2009) Structure-Property Relations for the Rheology of Dispersions of Charged Colloids. Ind. Eng. Chem. Res. 48:2380-2386.

52. Santamara-Holek I, Mendoza CI (2010) The rheology of concentrated suspensions of arbitrarily-shaped particles. J Coll Interf Sci 346:118-126.

53. Schall P, Cohen I, Weitz DA, Spaepen F (2004)Visualization of dislocation dynamics in collidal crystals. Science 305:1944-1948.

54. Sherwood, J. D., (1980)The primary electroviscous effect in a suspension of spheres. J. Fluid Mech., 101:609629.

55. Sherwood, J. D.; Rubio-Hernandez, F. J.; Ruiz-Reina, E. (2000) The primary electroviscous effect: thin double layers and a Stern layer. J. Colloid Interface Sci., 228: 713.

56. Shih WH, Shih WY, Kim SI, Liu J, Aksay IA (1990) Scaling behavior of the elastic properties of colloidal gels. Phys Rev A 42:4772-4779.

57. Sollich P (1998) Rheological constitutive equation for a model of soft glassy materials. Phys Rev E 58:738-459.

58. Stickel JJ, Powell RL (2005) Fluid mechanics and rheology of dense suspensions. Annu Rev Fluid Mech 37:129-149.

59. Tabor D (1951) Hardness of Metals Oxford Academic Publishers, Oxford.

60. Trompette JL, Meireles M (2003) Ion-specific effect on the gelation kinetics of concentrated colloidal silica suspensions. J Colloid Interface Sci 263:522-527.

61. Trompette JL, Clifton MJ (2004) Influence of ionic specificity on the microstructure and the strength of gelled colloid silica suspensions. J Colloid Interf Sci. 276:475-482.

62. Tsenoglou C (1990) Scaling concepts in suspension rheology. J Rheol 34:1523. 
63. van de Ven T.G.M. (1989) Colloidal Hydrodynamics. Academic Press.

64. Wang BX, Zhou LP, Peng XF (2003) A fractal model for predicting the effective thermal conductivity of liquid with suspensions of nanoparticle. Intern J Heat Mass Tranf 46:2665-2672

65. Yanez JA, Laarz E, Bergstrm L (1999) Viscoelastic Properties of Particle Gels. J Colloid Interf Sci. 209:162-172.

66. Zarzycki J (1988) Critical stress intensity factors of wet gels, J. Non-Cryst. Solids, 100:359-363. 